\title{
PTH-097 TRANSFERRING CARE OF HEPATITIS B-INFECTED MOTHERS FROM OBSTETRICS TO HEPATOLOGY: WHERE ARE THE BARRIERS?
}

doi:10.1136/gut.2011.239301.498

P Orchard, ${ }^{1, *}$ A Baxter, ${ }^{1}$ A McEwan, ${ }^{2}$ S D Ryder, ${ }^{1}$ M W James ${ }^{1}{ }^{1}$ Nottingham NIHR Biomedical Research Unit, Nottingham, UK; ${ }^{2}$ Department of Obstetrics, Nottingham University Hospitals, Nottingham, UK

Introduction Routine screening for HBV infection is performed during pregnancy and is identified more frequently with immigration from endemic areas. In addition to ensuring neonatal vaccination, $\mathrm{HBV}$-infected women subsequently require assessment, long-term monitoring or treatment. The authors examined their referral pathway and transfer of care between obstetrics and hepatology services.

Methods All women with HBV infection were identified from obstetric records from July 2008 to July 2009. Initial HBV screening bloods (HBVsAg) during pregnancy were sent to the regional Blood Transfusion Service. Confirmatory HBV testing, including quantitative PCR, was performed locally. Patient demographics and liver biochemistry were collected. Hepatology appointments offered, attendance and subsequent management were recorded. The authors aimed to identify barriers to patient identification and transfer of care for ongoing hepatology care.

Results Over 5000 pregnant women were screened for HBV infection during the study period. Communication of HBVpositive results from regional Blood Transfusion Laboratories varied between general practice and hospital obstetric departments. $41 \mathrm{HBVsAg}$ positive women were identified from local obstetric records $(0.8 \%$ of all screened). None originated from the UK; 14 (34.1\%) were African origin, 13 (31.8\%) Chinese, and the remaining 14 (34.1\%) from Eastern Europe, Middle East or Asia. 16 (39\%) were new diagnoses. 34 (83\%) had repeated confirmatory HBVsAg and/or HBV DNA testing performed locally. Median (range) HBV DNA $2.9 \times 10^{2}\left(0-1.9 \times 10^{8}\right.$ $\mathrm{IU} / \mathrm{ml}$ ), and $95 \%$ were eAg negative. Liver biochemistry was assessed in $36(88 \%)$ and was normal in 23 (64\%). Mean ALT was $25.1 \mathrm{IU} / 1$ (range $6-110$. Of 41 cases, referral to hepatology occurred in 34 (83\%), 32 (78\%) were offered a hepatology clinic appointment and $28(68 \%)$ of patients actually attended. One patient received treatment (lamivudine/tenofovir combination therapy).

Conclusion Almost 1\% of all pregnant women screened were HBV positive. Almost all were HBV eAg-negative and all originated from outside the UK. Improved communication of positive HBVsAg results from regional laboratories may improve identification of women requiring assessment. Over $30 \%$ HBV positive women identified from pregnancy screening are not assessed in hepatology clinics. Barriers to assessment include inadequate administration of patient transfer, language difficulties and poor understanding of the importance of monitoring and treatment of chronic hepatitis B infection.

Competing interests None.

Keywords hepatitis B infection, pregnancy, screening. 